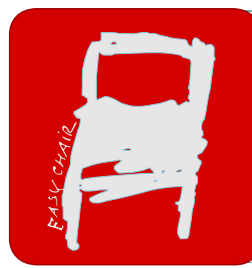

EPiC Series in Health Sciences

CAOS 2017. 17th Annual Meeting of the International Society for Computer Assisted Orthopaedic Surgery

\title{
Immersive Simulation of the Reduction of Femoral Diaphyseal Fracture
}

\author{
Mohamed Mediouni ${ }^{1}$, Djemel Ziou ${ }^{1}$, and François Cabana ${ }^{2}$ \\ 1 Département d'informatique, faculté des sciences, Université de Sherbrooke, Sherbrooke, Québec, \\ J1K 2R1, Canada \\ Mohamed.Mediouni@Usherbrooke.ca, Djemel.Ziou@Usherbrooke.ca \\ 2 Département de chirurgie, faculté de médecine et des sciences de la santé, Université de Sherbrooke, \\ Sherbrooke, Québec, J1H 5N4, Canada \\ Francois.Cabana@USherbrooke.ca
}

\begin{abstract}
With the advancement of the virtual technologies, three-dimensional surgical simulators are now possible. In this article, we describe an immersive simulation platform, allowing students in orthopedic surgery to learn how to deal with a sample diaphyseal fracture of the femur using LC-DCP plate hole, cortical screw and verbrugge forceps. To reach certain realism, weight of the objects and force feedback are used in addition to the visual scene and the 3D sound. The students feel the weight, the strength of the bone when they pierce the holes, and the vibration of the drill. The simulation is implemented by using CAVE, the CyberGlove, CyberGrasp, and 3D sound system.
\end{abstract}

\section{Introduction}

In orthopedic surgery, several models are used for training of surgeon learners, such as plastic and cadaveric models. However, the cost and the limited number of these models are the principal constraints. A possible alternative can be virtual reality (VR) based simulation. Some models based on virtual reality have been developed. Blyth et al. [2] developed a simulationbased training system for hip fracture fixation based on two-dimensional radiographic images to guide fracture reduction and implant placement using conventional computer interfaces (mouse and keyboard). The simulator performed by Mabrey et al. [6] aims to execute a proper examination of the knee and shaving a torn meniscus using computer (Silicon Graphics (SGI)) for visualization and feedback technology (PHANToM). Froelich et al. [5] use a phantom stylus that had an interface with force feedback to examine a potential model of evaluation of residents in the placement of guide wire during the fixation of femur fracture. This example in literature confirms that the virtual reality simulation is an effective tool to practice orthopedic surgery. However, they are devoted to concerns mainly the learning of arthroscopic knee and shoulder. There are many advantages of teaching using virtual simulator because the basic skills are acquired by the students in a secured way, and the use of the visualisation technologies enables specialists to acquire skills while minimizing cost, time, and the risk of error during surgery 
operation. However, the VR based simulations should fulfil requirements about the realism, the immersion and the latency. For example, it was pointed out that human learns more efficiently is an immersive environment $[4,1]$. In this work, we propose a VR based simulation diaphyseal fracture of femur which is different from others simulators in term of technologies used.

\section{Materials and Methods}

The aim of our VR simulation is to reduce a simple diaphyseal fracture of the femur using the appropriate instruments including LC-DCP plate 8 holes, cortical screws, hexagonal screwdriver, and pliers reduction (verbrugge forceps). It should also make it possible to evaluate the learner's achievements according to criteria established in the field of orthopedic surgery. In this work, we will develop a 3D operating room which contains a table of instruments, LC-DCP plate 8 holes, cortical screws, hexagonal screwdriver, verbrugge forceps and another table which contains a fixed bone. The surgeon can interact with all instruments described above in order to reduce the fracture. The conception and implantation of operating room is summarized as follows. A 3D model of femur is download from 3D CAD BROWSER and all surgical instruments were modelled using the geometry features of software Abaqus which are characterized by polygonal mesh. In fact, these models have a topological defects such as hole and separated regions. Consequently, the obtained mesh which represents 3D object has incomplete data. For that purpose, a step of preprocessing based on the method of Borodin et al. [3] has been implemented to overcome this limitation.

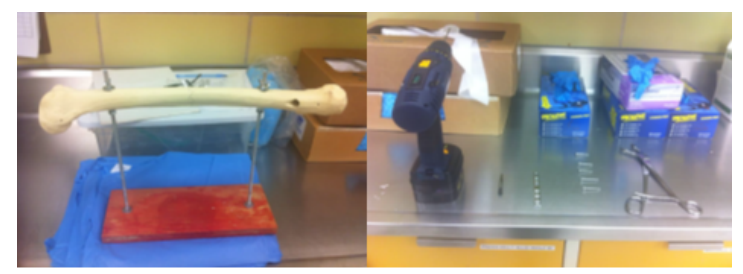

Figure 1: Sawbone and surgical instruments (Courtesy: Département de chirurgie, faculté de médecine et des sciences de la santé, Université de Sherbrooke, Québec, Canada)

The interactions with the preprocessed objects are carried out by using a CAVE consisting of four screens, four rear projectors, active stereoscopic system, 3D sound system, and haptic devices. For visual interaction, the user wears stereoscopic glasses which are equipped with passive markers to detect his position in the virtual environment and navigate in the virtual scene using joystick (see figure 2). The haptic interaction is implemented by using: (1) CyberGlove which follows from the position of the fingers in the virtual environment. (2) CyberGrasp which represents an exoskeleton that are positioned around the hands and provide a resistive force feedback to each finger. This device is primarily used to give the user the feeling of contact and blocking of the object by the fingers. (3) CyberForce which provides force feedback for hands and arms. CyberTrack which follows the orientation and the position of wrists of the user (see figure 2). We used the software library Virtualhand SDK to implement the kinesthetic sensory to detect all contact of virtual hand and 3D object in the CAVE. The calibration of glove is performed using software provided by CyberGlove Systems. The calibration process includes all finger in this order: pinky finger, ring finger, middle finger, and index finger. The CAVE is equipped with eight loudspeakers which allows the user to hear the sound of drill from different directions. We used the software library Fmod to implement 3D sound. 


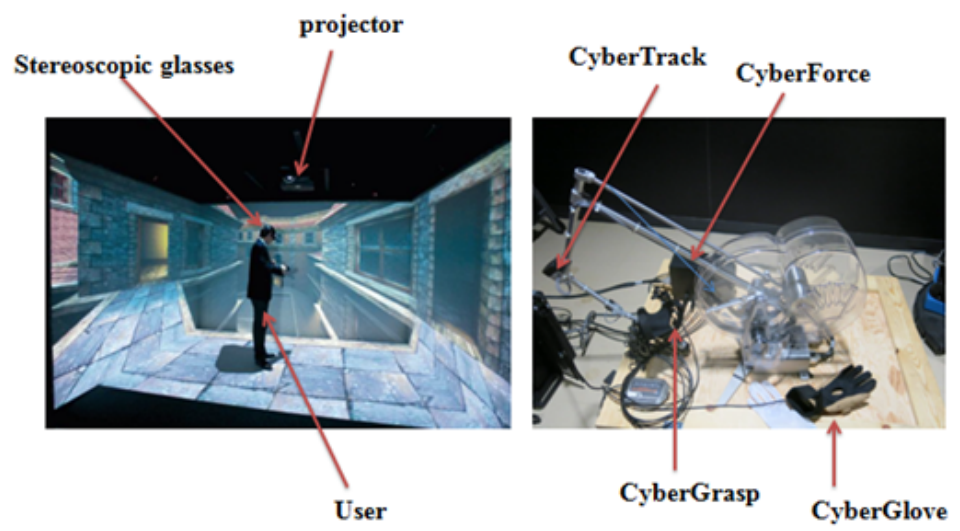

Figure 2: CAVE and haptic system

Within the CAVE, the learner can make gestures unconsciously like escaping a surgical instrument. In the referential $(\vec{X}, \vec{Y}, \vec{Z})$ fixed at the center of CAVE, we consider a surgical instrument placed on the table of instruments which has a position $x_{0}$ at time $(t=0)$. The surgeons move the instrument according to the trajectory $x(t)$. The position and the velocity of instrument are given by CyberTrack. At the time $t$, the instrument dropped into space under its weight. In this case, the instrument undergoes a trajectory given by this equation: $x(t)=\frac{1}{2} g^{2} t+v t+x$, where $v=0$ is the initial velocity, $x$ is the initial position and $g$ is the gravity. The velocity is calculated with this equation: $v(t)=\frac{d x(t)}{t}$. During the fall, the instrument can collide one or many objects. To simplify the collision detection, each objet is englobed in the bounding sphere. If $x_{1}$ and $x_{2}$ are the centre of spheres and $r_{1}$ and $r_{2}$ are their radii, then a contact point is a point such that it verifies the equations of the two spheres: $S=\| x_{1}-x_{2}||-\left(r_{1}+r_{2}\right)$, where $\|$.$\| is the Euclidean. If S=0$, then a contact is declared. In addition, at the point of contact, a high force of short duration which have referred to shock force pushes the instrument to move away in the space and undergoes another trajectory calculated by the library bullet.

To make a holes on the bone, a collision between drill bit and bone is detected by the same rule $\mathrm{S}$ described above. At the point of contact, an algorithm is executed to delete the intersection surface of the drill bit inside the bone which is implemented by the library GTS. At the same time, to feel the vibration during drilling, a haptic model is proposed. An appropriate force is reflected back to the user which is estimated and applied by using CyberGrasp and the library Virtualhand SDK. In addition, a force $F$ must be exerted on the bone for vibration. According to Zilles et al. [7], two visual reference points are added to the bone that are Haptic Interaction Point $(H I P)$ which is defined as the end of the drill bit and the God Object $(G O)$ point which is placed on the surface of the bone (see figure 4). By using a spring model, the force is determined with this equation: $F=k(H I P-G O)$, where $k$ is the spring stiffness which has been set $1.5 \mathrm{~N} / \mathrm{mm}$ for trabecular bone and $3.0 \mathrm{~N} / \mathrm{mm}$ for cortical bone. 


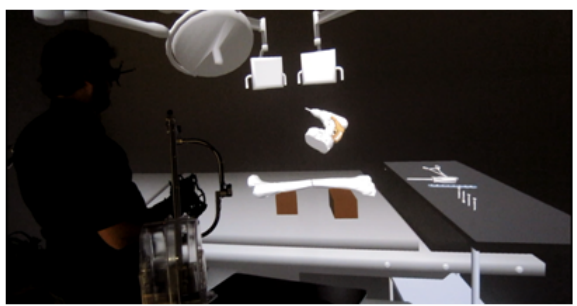

Figure 3: Operating room in the CAVE in which the user interacts with surgical instruments

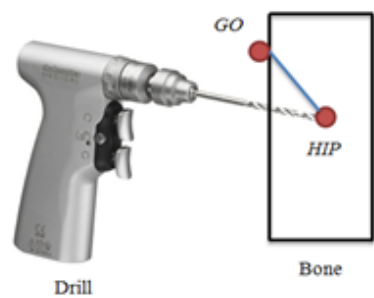

Figure 4: Haptic simulation

\section{Results}

In this work, we have developed a 3D operating room in the CAVE, where 3D objects are placed contextually according to the relationship between them and the further usage. The surgeon interacts with an avatar which represents his hand in the CAVE. Developing a physical engine in the CAVE help to increase the realism and immersion to practice surgery (see figure 5)

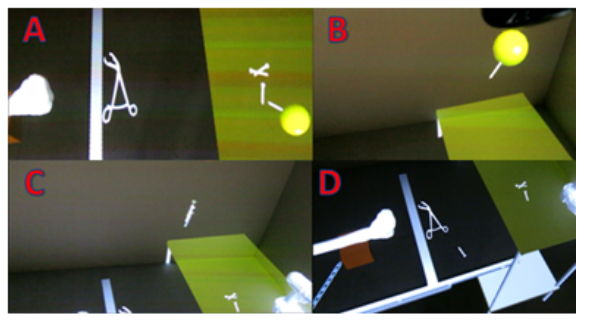

Figure 5: (A) The selection of the screw from the table of surgical instruments, (B) the surgeon moves the screw in the space, $(\mathrm{C})$ the surgeon looses the screw, (D) the screw falls on the table of operation.

\section{Discussion}

As we have seen that the technique of virtual reality gives user a 3D simulation close to reality. In the CAVE, we have developed 3D operating room which contains all surgical instruments. 3D sound is synchronized with 3D images which give user a controllable and comfortable simulation to practice surgery. In addition, validating the simulation with students remains an essential 
step to study their perceptions toward this virtual experience. The successful of our orthopedic simulation will help others surgeons in other fields of surgery to follow the same path for training.

\section{Acknowledgments}

The completion of this article could not have been possible without the participation and assistance of many people. The authors would like to express their appreciations and indebtedness particularly to Jérôme Couture, Patricia Sirois, Daniel Lévesque and Patrick Bellavance Marcoux.

\section{References}

[1] Hannouche D Nizard R Aim F, Lonjon G. Effectiveness of virtual reality training in orthopaedic surgery. Arthroscopy: The Journal of Arthroscopic and Related Surgery, 32(1):224-232, 2016.

[2] Anderson IA Blyth P, Stott NS. A simulation-based training system for hip fracture fixation for use within the hospital environment. Injury, 38(10):1197-1203, 2007.

[3] Klein R Borodin P, Novotni M. Progressive gap closing for mesh repairing, advances in modelling, animation and rendering. In Computer Graphics Society, 2002.

[4] Ozell B Labelle H Aubin CE Cote M, Boulayl JA. Virtual reality simulator for scoliosis surgery training: Transatlantic collaborative tests. In IEEE International Workshop on Haptic Audio Visual Environments and their Applications Ottawa- Canada, 2008.

[5] Novicoff WM Saleh KJ Allan DG Froelich JM, Milbrandt JC. Surgical simulators and hip fractures: a role in residency training? J Surg Educ, 68(4):298-302, 2011.

[6] Kasser JR Sweeney HJ Zarins B Mevis H Garrett WE Jr Poss R Cannon WD Mabrey JD, Gillogly SD. Virtual reality simulation of arthroscopy of the knee. Arthroscopy: The Journal of Arthroscopic and Related Surgery, 18(6):28, 2002.

[7] Salisbury JK Zilles CB. A constraint-based god-object method for haptic display. In Proc. of the IEEE Conference on Intelligent Robots and Systems, 1995. 